\title{
Cosmology with Relativistically Varying Physical Constants
}

\author{
Rajendra P. Gupta \\ Department of Physics, University of Ottawa, Ottawa, Canada K1N 6N5 \\ rgupta4@uottawa.ca
}

(Dated: June 25, 2020)

\begin{abstract}
We have shown that our varying physical constant model is consistent with the recently published variational approach wherein Einstein equations are modified to include the variation of the speed of light $c$, gravitational constant $G$ and cosmological constant $\Lambda$ using the Einstein-Hilbert action. The general constraint resulting from satisfying the local conservation laws and contracted Bianchi identities provides the freedom to choose the form of the variation of the constants as well as how their variations are related. When we choose $\dot{G} / G=\dot{3} c / c$, the same as in our quasi-phenomenological model, $c=$ $c_{0} \exp \left[\left(a^{\alpha}-1\right)\right], G=G_{0} \exp \left[3\left(a^{\alpha}-1\right)\right]$ and $\Lambda=\Lambda_{0} \exp \left[\left(a^{-\alpha}-1\right)\right]$, where $a$ is the scale factor and $\alpha=$ 1.8 , we are able to confirm the success of our the model in explaining three astrometric anomalies and the null results on the variation of $G$ and the fine structure constant. We show that the model: (a) fits the supernovae 1a observational data better than the $\Lambda \mathrm{CDM}$ model; (b) determines the first peak in the power spectrum of the cosmic microwave background temperature anisotropies at multipole value of $l=217.3$; (c) calculates the age of the universe as $14.1 \mathrm{Gyr}$; and (d) finds the BAO acoustic scale to be $145.2 \mathrm{Mpc}$. These numbers are within less than $3 \%$ percent of the observed values and the values obtained by the $\Lambda \mathrm{CDM}$ model. Surprisingly we find that the dark-energy density is negative in a universe that has significant negative curvature and whose expansion is accelerating at a faster rate than predicted by the $\Lambda$ CDM model.
\end{abstract}

Keywords: cosmology theory; cosmological parameters; distances and redshifts; variable physical constants; VSL; CMB; BAO.

\section{Introduction}

Despite the stunning success of the standard model in explaining the cosmological observations, there remain many gaps that need to be bridged. We believe these gaps warrant looking at alternative theories based on examining the foundation of physics that has been developed over centuries from local observations extrapolated to the universe at large. One of the foundations is that constants relating various observables are indeed constants everywhere and at every time in the universe. This is especially so because attempts to measure their variation has put very tight limit on their potential variation.

Varying physical constant theories have been in existence since time immemorial ${ }^{1-3}$ especially since Dirac $^{4,5}$ in 1937 suggested variation of the constant $G$ based on his large number hypothesis. Brans and Dicke ${ }^{6}$ developed the $G$ variation theory compliant with general relativity in which constant $G$ was raised to the status of scalar field potential. While Einstein developed his ground breaking theory of special relativity based on the constancy of the speed of light, he did consider its possible variation ${ }^{7}$. This was followed by the varying speed of light theories by Dicke ${ }^{8}$, Petit ${ }^{9}$, Moffatt ${ }^{10,11}$. More recently, Albrecht and Magueijo ${ }^{12}$ and Barrow ${ }^{13}$ developed such a theory in which Lorentz invariance is broken as there is a preferred frame in which scalar field is minimally coupled to gravity. Other proposals include locally invariant theories ${ }^{14,15}$ and vector field theories that cause spontaneous violation of Lorentz invariance ${ }^{16}$.

The most comprehensive review of the varying fundamental physical constants was done by Uzan $^{17}$ in 2003 followed by his more recent review ${ }^{18}$. Chiba ${ }^{19}$ has provided an update of the observational and experimental status of the constancy of physical constants. We therefore will not attempt to cover the subject's current status except to mention a few of direct relevance to this work. 
Variable physical constants are introduced in most of the proposed theories at the cost of either not conserving energy-momentum or violating Bianchi identity, which then leads to breaking the covariance of the theory. Such theories may be considered inconsistent or ad hoc ${ }^{20}$ or quasi-phenomenological21. An action principle to take into account the variation of the fundamental constants that are being considered in a theory is required for generalization of Einstein equations. Costa et al..$^{22,23}$ have attempted this approach by considering the speed of light $c$, gravitational constant $G$, and cosmological constant $\Lambda$ as scalar fields, and introducing an Einstein-Hilbert action that is considered consistent with the Einstein equations as well as with the general constraint they introduced that is compliant with contracted Bianchi identities and standard local conservation laws. Their approach is general covariant as it preserves the invariance of the general relativity. However, here we specify that the action formulation of this paper 22,23 is not a correct one. With the standard Einstein-Hilbert action, when one promotes $G$ and $c$ as scalar fields, the Ricci-scalar in the action gets "non-minimally coupled" with the scalar fields. Therefore, the modified Einstein-Hilbert action becomes an action of scalar-tensor theory of gravity, which, using the variational principal, does not yield Einstein's equation. This issue has been discussed in literature ${ }^{20}$. However, in the work of Costa et al.22,23, the focus was to explain early universe problems - the flatness problem and the horizon problem - without invoking inflation, which they have successfully shown by simply promoting the constants $G$ and $c$ in Einstein's equation as scalar fields. Here we shall follow the same prescription. However, we will limit ourselves to consider important direct cosmological observations from now up to the time of cosmic microwave background emission. An action formulation of such modified Einstein's equation is kept as a future work for the time being.

We will begin with establishing the theoretical background for our work in this paper in Section 2 starting from the equations presented in Costa et al.'s paper ${ }^{22}$. We will confine ourselves to two considerations of the model: one with varying cosmological constant, and another with fixed cosmological constant. The standard formulae used in cosmology are based on fixed physical constants. We will go through carefully the derivations of those we will use and determine how they are modified when the constants are allowed to evolve. These involve generalization of the luminosity distance, and therefore also of the distance modulus, as well as the scale factor for the surface of last scattering and the deceleration parameter.

Section 3 delineates the results. Firstly, we present the parameters and curves obtained by fitting the redshift versus distance modulus Pantheon Sample data ${ }^{24}$ for 1048 supernovae 1a to the $\Lambda$ CDM model and the two variable constants models. All the models yield very good fit to the data but with different parameters. This indicates SNe Ia data fit is necessary but not the deciding consideration for selecting a model. The second test is to see how well a model computes the multipole moment of the first peak in the power spectrum due to the temperature anisotropies in the cosmic microwave background. The third test may be considered how well the models match the value of the BAO acoustic scale obtained from the peak in the two point separation correlation function determined from measurements over a million galaxies. Another important test comprises determining the age of the universe.

Section 4 discusses the findings of this paper and Section 5 presents our conclusions.

\section{Theoretical Background}

Following Costa et al. 22 we may write the Einstein equations with varying physical constants (VPC) with respect to time $t$ - speed of light $c=c(t)$, gravitational constant $G=G(t)$ and cosmological constant $\Lambda=\Lambda(t)$ - applicable to the homogeneous and isotropic universe, as follows:

$$
G_{\mu \nu}=\left(\frac{8 \pi G(t)}{c(t)^{4}}\right) T_{\mu \nu}-\Lambda(t) g_{\mu \nu}
$$


Here $G_{\mu \nu}=R_{\mu \nu}-\frac{1}{2} g_{\mu \nu} R$ is the Einstein tensor with $R_{\mu \nu}$ the Ricci tensor and $R$ the Ricci scalar, and $T_{\mu \nu}$ is the stress energy tensor. Applying the contracted Bianchi identities, torsion free continuity and local conservation laws

$$
\nabla^{\mu} G_{\mu \nu}=0 \text { and } \nabla^{\mu} T^{\mu \nu}=0
$$

one gets a general constraint equation for the variation of the physical constants

$$
\left[\frac{1}{G} \partial_{\mu} G-\frac{4}{c} \partial_{\mu} c\right]\left(\frac{8 \pi G}{c^{4}}\right) T^{\mu v}-\left(\partial_{\mu} \Lambda\right) g^{\mu \nu}=0
$$

Now the FLRW (Friedmann-Lemaitre-Robertson-Walker) metric for the geometry of the universe is written as:

$$
d s^{2}=-c^{2}(t) d t^{2}+a^{2}(t)\left[\frac{1}{1-k r^{2}} d r^{2}+r^{2}\left(d \theta^{2}+\sin ^{2} \theta d \phi^{2}\right)\right]
$$

with $k=-1,0,1$ depending on the spatial geometry of the universe.

The stress-energy tensor, assuming that the universe content can be treated as perfect fluid, is written as:

$$
T^{\mu \nu}=\frac{1}{c^{2}(t)}(\varepsilon+p) U^{\mu} U^{\nu}+p g^{\mu \nu} .
$$

Here $\varepsilon$ is the energy density, $p$ is the pressure, $U^{\mu}$ is the 4-velocity vector with the constraint $g_{\mu \nu} U^{\mu} U^{\nu}=$ $-c^{2}(t)$. (Unless necessary to avoid confusion, we will drop showing $t$ variation, e.g. $c(t)$ is written as $c$.)

Solving the Einstein equation (such as by using Maplesof ${ }^{25}$ ) then yields VPC compliant Friedmann equations:

$$
\begin{gathered}
H^{2} \equiv \frac{\dot{a}^{2}}{a^{2}}=\frac{8 \pi G \varepsilon}{3 c^{2}}+\frac{\Lambda c^{2}}{3}-\frac{k c^{2}}{a^{2}}, \Rightarrow \dot{a}^{2}=a^{2}\left(\frac{8 \pi G \varepsilon}{3 c^{2}}+\frac{\Lambda c^{2}}{3}-\frac{k c^{2}}{a^{2}}\right), \\
\frac{\ddot{a}}{a}=-\frac{4 \pi G}{3 c^{2}}(\varepsilon+3 p)+\frac{\Lambda c^{2}}{3}+\frac{\dot{c}}{c} \frac{\dot{a}}{a}=-\frac{4 \pi G}{3 c^{2}}(\varepsilon+3 p)+\frac{\Lambda c^{2}}{3}+\frac{\dot{c}}{c} \frac{a}{\dot{a}} H^{2} .
\end{gathered}
$$

Taking time derivative of Eq. (6), dividing by $2 a \dot{a}$ and equating it with Eq. (7), yields the general continuity equation:

$$
\dot{\varepsilon}+3 \frac{\dot{a}}{a}(\varepsilon+p)=-\left[\left(\frac{\dot{G}}{G}-4 \frac{\dot{c}}{c}\right) \varepsilon+\frac{c^{4}}{8 \pi G} \dot{\Lambda}\right]
$$

Eq. (3) for the FLRW metric and perfect fluid stress-energy tensor reduces to:

$$
\left[\left(\frac{\dot{G}}{G}-4 \frac{\dot{c}}{c}\right) \frac{8 \pi G}{c^{4}} \varepsilon+\dot{\Lambda}\right]=0
$$

therefore,

$$
\dot{\varepsilon}+3 \frac{\dot{a}}{a}(\varepsilon+p)=0
$$

Using the equation of state relation $p=w \varepsilon$ with $w=0$ for matter and $w=1 / 3$ for relativistic particles, the solution for this equation is $\varepsilon=\varepsilon_{0} a^{-3-3 w}$, where $\varepsilon_{0}$ is the current energy density of all the components of the universe when $a=a_{0}=1$. 
Next we need to consider the continuity equation Eq. (9). The simplest solution is by assuming $\Lambda=$ constant. Then $\frac{\dot{G}}{G}=4 \frac{\dot{c}}{c}$, i.e. $\frac{G}{G_{0}}=\frac{c^{4}}{c_{0}^{4}}$. This is what Costa et al. ${ }^{22}$ used in their paper. We will label this model as VcG model (varying $c$ and $G$ model). In fact they dropped the cosmological constant altogether for their $c$-flation solution. However, one could choose any relationship between $G$ and $c$, say $\frac{\dot{G}}{G}=\sigma \frac{\dot{c}}{c}$. Then from Eq. (9), by defining $\varepsilon_{\Lambda}=\frac{c^{4} \Lambda}{8 \pi G}$, we have

$$
\frac{8 \pi G}{c^{4}} \frac{\dot{c}}{c}(4-\sigma) \varepsilon=\dot{\Lambda}, \Rightarrow \frac{\dot{c}}{c}=\frac{c^{4} \Lambda}{8 \pi G}\left(\frac{\dot{\Lambda}}{\Lambda}\right)\left(\frac{1}{(4-\sigma) \varepsilon}\right) \equiv \frac{\varepsilon_{\Lambda}}{(4-\sigma) \varepsilon} \frac{\dot{\Lambda}}{\Lambda}, \Rightarrow \varepsilon_{\Lambda}=\frac{\dot{c}}{c} \frac{\Lambda}{\dot{\Lambda}}(4-\sigma) \varepsilon
$$

The parameter $\sigma$ may be determined based on the physics or by fitting the observations. We have determined in the past $\mathrm{t}^{21}$ that $\sigma=3$, i.e. $\frac{\dot{G}}{G}=3 \frac{\dot{c}}{c}$ and confirmed it by fitting the SNe 1a data. Thus, we must have $\varepsilon_{\Lambda}=\frac{\dot{c}}{c} \frac{\Lambda}{\dot{\Lambda}} \varepsilon$. We will label this model as $\operatorname{VcG} \Lambda$ model (varying $c, G$ and $\Lambda$ model).

The most common way of defining the variation of the constant is by using the scale factor powerlaw ${ }^{26,27}$, such as $c=c_{0} a^{\alpha}$ which results in $\frac{\dot{c}}{c}=\alpha \frac{\dot{a}}{a}=\alpha H$. The advantage is that it results in very simple Freedmann equations. However, as $a \rightarrow 0$ the variable constant tends to zero or infinity depending on the sign of $\alpha$. So, it yields reasonable results when $a=\frac{1}{1+z}$ corresponds to relatively small redshift $z$, but not for large $z$. What we have used in the past ${ }^{21}$ is the relations like $\frac{\dot{c}}{c}=\alpha H_{0}$, i.e. $c=$ $c_{0} \exp \left[\alpha H_{0}\left(t-t_{0}\right)\right]$, which leads to a limiting value of $c$ at $t=0$. This approach was very useful when solving problems with $t-t_{0}$ very small, such as for explaining astrometric anomalies. However, due to the involvement of time coordinate, it is difficult to use it in the Friedmann equations for their general solution. We therefore tried another relation that results in:

$$
c=c_{0} \exp \left[\left(a^{\alpha}-1\right)\right], G=G_{0} \exp \left[3\left(a^{\alpha}-1\right)\right], \text { and } \Lambda=\Lambda_{0} \exp \left[\left(a^{\beta}-1\right)\right]
$$

Their limitation is that the constants can increase or decrease in the past at most by a factor of $e=2.7183$ (within the region of their applicability) in the case of $c$ and $\Lambda$, and by a factor of $e^{-3}$ for the case of $G$. In the limit of $a \rightarrow 1$, they reduce to the earlier exponential forms. For example $\dot{c}=c_{0} \exp \left(a^{\alpha}-1\right) \times \alpha a^{\alpha-1} \dot{a}$, or $\frac{\dot{c}}{c}=\alpha a^{\alpha} \frac{\dot{a}}{a^{\prime}}$, which in the limit of $a \rightarrow 1$ is $\frac{\dot{c}}{c}=\alpha H_{0}$, or $c=c_{0} \exp \left[\alpha H_{0}\left(t-t_{0}\right)\right]$. It should be mentioned that we found in an earlier work ${ }^{28}$ that analytically $\alpha=1.8$.

Using relations of Eq. (12), we can now write Eq. (11) for $\sigma=3$,

$$
\frac{c^{4} \Lambda}{8 \pi G} \equiv \varepsilon_{\Lambda}=\frac{\dot{c} \Lambda}{c} \frac{\Lambda}{\dot{\Lambda}} \varepsilon=\frac{\alpha}{\beta} a^{\alpha-\beta} \varepsilon
$$

The first Friedmann equation, Eq. (6), becomes

$$
H^{2}=\frac{8 \pi G}{3 c^{2}}\left(\varepsilon+\frac{\Lambda c^{4}}{8 \pi G}\right)-\frac{k c^{2}}{a^{2}}=\frac{8 \pi G}{3 c^{2}}\left(\varepsilon+\varepsilon_{\Lambda}\right)-\frac{k c^{2}}{a^{2}}=\frac{8 \pi G}{3 c^{2}} \varepsilon\left(1+\frac{\alpha}{\beta} a^{\alpha-\beta}\right)-\frac{k c^{2}}{a^{2}} .
$$

Dividing by $H_{0}^{2}$, and since $\varepsilon=\varepsilon_{m}+\varepsilon_{r}=\varepsilon_{m, 0} a^{-3}+\varepsilon_{r, 0} a^{-4}$ where subscript $m$ is for matter and $r$ is for radiation (relativistic particles, e. g. photons and neutrinos), we get

$$
\frac{H^{2}}{H_{0}^{2}}=\frac{8 \pi G}{3 c^{2} H_{0}^{2}}\left(\varepsilon_{m, 0} a^{-3}+\varepsilon_{r, 0} a^{-4}\right)\left(1+\frac{\alpha}{\beta} a^{\alpha-\beta}\right)-\frac{k c^{2}}{H_{0}^{2} a^{2}} .
$$

At $t=t_{0}$ (current time), $a=1$ and $H=H_{0}$. Therefore, 


$$
1=\frac{8 \pi G_{0}}{3 c_{0}^{2} H_{0}^{2}}\left(\varepsilon_{m, 0}+\varepsilon_{r, 0}\right)\left(1+\frac{\alpha}{\beta}\right)-\frac{k c_{0}^{2}}{H_{0}^{2}}=\left(\Omega_{m, 0}+\Omega_{r, 0}\right)\left(1+\frac{\alpha}{\beta}\right)-\frac{k c_{0}^{2}}{H_{0}^{2}} .
$$

Here we have defined the current critical density as $\varepsilon_{c, 0}=3 c_{0}^{2} H_{0}^{2} / 8 \pi G_{0}, \Omega_{m, 0}=\varepsilon_{m, 0} / \varepsilon_{c, 0}$ and $\Omega_{r, 0}=$ $\varepsilon_{r, 0} / \varepsilon_{c, 0}$. Thus, by defining $\Omega_{0}=\left(\Omega_{m, 0}+\Omega_{r, 0}\right)\left(1+\frac{\alpha}{\beta}\right)$, we may write Eq. (16)

$$
\begin{gathered}
\Omega_{k, 0} \equiv-\frac{k c_{0}^{2}}{H_{0}^{2}}=1-\Omega_{0}, \\
\frac{H^{2}}{H_{0}^{2}}=\exp \left[\left(a^{\alpha}-1\right)\right]\left(\Omega_{m, 0} a^{-3}+\Omega_{r, 0} a^{-4}\right)\left(1+\frac{\alpha}{\beta} a^{\alpha-\beta}\right)+\Omega_{k, 0} \exp \left[2\left(a^{\alpha}-1\right)\right] a^{-2} \equiv E(a)^{2} .
\end{gathered}
$$

Now, the FLRW metric, Eq. (4), can be written in its alternative form ${ }^{29}$ as

$$
d s^{2}=-c^{2} d t^{2}+a(t)^{2}\left[d r^{2}+S_{k}(r)^{2}\left(d \theta^{2}+\sin ^{2} \theta d \phi^{2}\right)\right] .
$$

Here $S_{k}(r)=R \sin (r / R)$ for $k=+1$ (closed universe); $S_{k}(r)=r$ for $k=0$ (flat universe), $S_{k}(r)=$ $R \sinh (r / R)$ for $k=-1$ (open universe), where $R$ is the parameter related to the curvature. The proper distance $d_{P}$ between an observer and a source is determined at fixed time by following a spatial geodesic at constant $\theta$ and $\phi$. Then

$$
d s=a(t) d r \Rightarrow d_{P}(t)=a(t) \int_{0}^{r} d r=a(t) r
$$

We could determine $r$ following a null geodesic from the time $t$ a photon is emitted by the source to the time $t_{0}$ it is detected by the observer with $d s=0$ in Eq. (19) at constant $\theta$ and $\phi$ :

$$
c^{2} d t^{2}=a(t)^{2} d r^{2} \Rightarrow \frac{c d t}{a(t)}=d r \Rightarrow r=\int_{0}^{r} d r=\int_{t}^{t_{0}} \frac{c d t}{a(t)} \Rightarrow d_{P}=a\left(t_{0}\right) \int_{t}^{t_{0}} \frac{c d t}{a(t)}
$$

We may write $d t=d a \cdot d t / d a=d a / \dot{a}=d a / a \dot{a} / a=d a / a H$, and $a=1 /(1+z), d a=-d z /(1+z)^{2}$ $=-a^{2} d z$. Therefore

$$
\begin{gathered}
d t=-\frac{a d z}{H}=-\frac{a d z}{\frac{H_{0} H}{H_{0}}}=-\frac{a d z}{H_{0} E(a)}, \text { and } \\
d_{P}=\frac{1}{H_{0}} \int_{0}^{Z} \frac{c d z}{E(z)}=\frac{c_{0}}{H_{0}} \int_{0}^{Z} \frac{\exp \left[\left((1+z)^{-\alpha}-1\right)\right] d z}{E(z)} .
\end{gathered}
$$

Here $E(z)$ is obtained from Eq. (18) by substituting $1 /(1+z)$ for $a$.

Costa et al. Model: Let us also consider the proposition of Costa et al..$^{22}$ that $\Lambda$ be taken constant - the VcG model. While they did not propose how $c$ and $G$ should vary in general, we will use the same form as for the $\mathrm{VcG} \Lambda$ model so that the results of the two can be comparable. In that case, as mentioned above, we have

$$
c=c_{0} \exp \left[\left(a^{\alpha}-1\right)\right], G=G_{0} \exp \left[4\left(a^{\alpha}-1\right)\right], \text { and } \Lambda=\Lambda_{0} .
$$

Then, the first Friedmann equation becomes:

$$
H^{2}=\exp \left[2\left(a^{\alpha}-1\right)\right]\left[\frac{8 \pi G_{0}}{3 c_{0}^{2}}\left(\varepsilon_{m, 0} a^{-3}+\varepsilon_{r, 0} a^{-4}\right)+\frac{\Lambda_{0} c_{0}^{2}}{3}-k c_{0}^{2} a^{-2}\right]
$$

Then, as before, we get 


$$
\frac{H^{2}}{H_{0}^{2}}=\exp \left[2\left(a^{\alpha}-1\right)\right]\left[\Omega_{m, 0} a^{-3}+\Omega_{r, 0} a^{-4}+\Omega_{\Lambda, 0}+\Omega_{k, 0} a^{-2}\right] \equiv E(a)^{2}
$$

Substituting $a=1 /(1+z)$ we get $E(z)^{2}$. We can use Eq. (23) with this $\mathrm{E}(\mathrm{z})$ to obtain proper distance corresponding to Costa et al. model VcG.

$\Lambda C D M$ Model: If we substitute $\alpha=0$ in Eq. (26), we get back the invariant physical constants case. We can then solve for the standard $\Lambda \mathrm{CDM}$ model parameters by fitting the observational data, or put the parameter of our choice to see how well they fit the data.

\subsection{Redshift vs. Distance Modulus}

We will now consider the specifics of applying the theory to develop relationship between the redshift $z$ and distance modulus $\mu$. First of all we will check if the relation $a=1 /(1+z)$ holds when physical constants are varying.

Since light travels along null geodesics, we have as per Eq. (21) $c^{2} d t^{2}=a(t)^{2} d r^{2}$, or $d r=c d t / a(t)$. A wave crest of the light emitted from a galaxy at time $t_{e}$ and observed at time $t_{0}$ travels a distance

$$
r=\int_{0}^{r} d r=\int_{t_{e}}^{t_{0}} \frac{c d t}{a(t)}
$$

Next wave crest will be emitted at time $t_{e}+\lambda_{e} / c_{e}$ and observed at time $t_{0}+\lambda_{0} / c_{0}$ and will cover the same distance

$$
r=\int_{0}^{r} d r=\int_{t_{e}+\lambda_{e} / c_{e}}^{t_{0}+\lambda_{0} / c_{0}} \frac{c d t}{a(t)}
$$

Therefore,

$$
\int_{t_{e}}^{t_{0}} \frac{c d t}{a(t)}=\int_{t_{e}+\lambda_{e} / c_{e}}^{t_{0}+\lambda_{0} / c_{0}} \frac{c d t}{a(t)}
$$

Subtracting from both sides the integral $\int_{t_{e}+\lambda_{e} / c}^{t_{0}} \frac{c d t}{a(t)}$ we get

$$
\int_{t_{e}}^{t_{e}+\lambda_{e} / c_{e}} \frac{c d t}{a(t)}=\int_{t_{0}}^{t_{0}+\lambda_{0} / c_{0}} \frac{c d t}{a(t)}
$$

Assuming $c$ and $a(t)$ remain unchanged over the extremely short time between two consecutive wave crests compared to the age of the universe, we have

$$
\frac{c_{e}}{a_{e}} \int_{t_{e}}^{t_{e}+\lambda_{e} / c_{e}} d t=\frac{c_{0}}{a_{0}} \int_{t_{0}}^{t_{0}+\lambda_{0} / c_{0}} d t \Rightarrow \frac{c_{e}}{a_{e}}\left(\frac{\lambda_{e}}{c_{e}}\right)=\frac{c_{0}}{a_{0}}\left(\frac{\lambda_{0}}{c_{0}}\right) \Rightarrow \frac{\lambda_{e}}{a_{e}}=\frac{\lambda_{0}}{a_{0}}
$$

Since $z \equiv\left(\lambda_{0}-\lambda_{\mathrm{e}}\right) / \lambda_{e}$, we get from above $1+z=a_{0} / a_{e}=1 / a_{e}$ with $a_{0} \equiv 1$. This result is the same as for the case when $c$ is invariant.

Now the distance of a source is determined by measuring flux of radiation arriving from the source with known luminosity. We therefore need to relate proper distance $d_{P}$ that relates to the redshift $z$ as per Eq. (23) to the luminosity distance $d_{L}$ which relates to the flux. We will then be able to establish what we mean by luminosity distance in the variable physical constant approach and how it differs from the case when constants are indeed constants.

The photons emitted by a source at time $t_{e}$ are spread over the sphere of proper radius $S_{k}\left(d_{P}\right)$ and, referring to Eq. (19), proper surface area $A_{P}\left(t_{0}\right)$ is given by ${ }^{29}$ 


$$
A_{P}\left(t_{0}\right)=4 \pi S_{k}\left(d_{P}\right)^{2}
$$

The flux is defined as luminosity $L$ divided by the area in a stationary universe. When the universe is expanding then the flux is reduced by a factor $1+z$ due to energy reduction of the photons. We need to also determine how the increase in distance between the emitted photons affects the flux.

The proper distance between two emitted photons separated by a time interval $\delta t_{e}$ is $c_{e} \delta t_{e}$ whereas the proper distance between the same two photons when observed would become $\left(c_{0} \delta t_{e}\right)(1+z)$. Thus the proper distance would increase by a factor $\left(c_{0} \delta t_{e}\right)(1+z) / c_{e} \delta t_{e}=(1+z) c_{0} / c_{e}$. When $c$ is constant, i.e. $c_{e}=c_{0}$, this effect reduces the flux by a factor of $1+z$. But when it is not, there is an additional factor $c_{0} / c_{e}$ that we have to consider to correct the flux. We thus have to account for an extra factor $c_{0} / c_{e}$ in the luminosity distance increase when calculating proper distance of a source from its flux data.

The flux $f$ and the luminosity distance $d_{L}$ relation may therefore be written as

$$
\begin{gathered}
f=\frac{L}{4 \pi S_{k}\left(d_{P}\right)^{2}(1+z)^{2}\left(\frac{c_{0}}{c_{e}}\right)}, \\
d_{L} \equiv\left(\frac{L}{4 \pi f}\right)^{1 / 2}=S_{k}\left(d_{P}\right)(1+z)\left(\frac{c_{0}}{c_{e}}\right)^{1 / 2} .
\end{gathered}
$$

The distance modulus $\mu$ by definition is related to the luminosity distance $d_{L}$ :

$$
\mu \equiv 5 \log _{10}\left(\frac{d_{L}}{1 M p c}\right)+25=5 \log _{10} S_{k}\left(d_{P}\right)+5 \log _{10}(1+z)+25+2.5 \log _{10}\left(\frac{c_{0}}{c_{e}}\right)
$$

The last term is the extra correction term that must be included in calculating the distance modulus when one is considering the varying speed of light irrespective of the model applied and whether or not the space is flat.

\subsection{CMB Power Spectrum}

Here we will be focussing on the first peak in the power spectrum of the cosmic microwave background $(\mathrm{CMB})$ temperature anisotropies and the related parameters such as its multipole moment, sound horizon distance at the time universe became transparent, acoustic scale derived from baryonic acoustic oscillation, and angular diameter distance of the surface of last scattering.

The sound horizon distance $d_{s}\left(t_{l s}\right)$ is the distance sound travels at speed $c_{s}(t)$ in photon-baryon fluid from the big-bang until the time such plasma disappeared due to the formation of the atoms, i.e. the time of last scattering $t_{l s}$. Following Eq. (21) we may write 30

$$
d_{s h}\left(t_{l s}\right)=a\left(t_{l s}\right) \int_{0}^{t_{l s}} \frac{c_{s}(t) d t}{a(t)}
$$

The speed of sound $c_{s}(t)$ in terms of the speed of light $c(t)$ in the photon-baryon fluid with baryon density $\Omega_{b}$ and radiation density $\Omega_{r}$ is given by ${ }^{30}$ :

$$
c_{S}(t) \approx \frac{c(t)}{\sqrt{3}}\left(1+\frac{3 \Omega_{b}}{4 \Omega_{r}}\right)^{-1 / 2} .
$$

Substituting it in Eq. (36) and making use of Eq. (23), we get

$$
d_{s h}\left(z_{l s}\right)=\frac{c_{0}}{\sqrt{3} H_{0}\left(1+z_{l s}\right)} \int_{\infty}^{z_{l s}} \frac{\exp \left[\left((1+z)^{-\alpha}-1\right)\right] d z}{\left(1+\frac{3 \Omega_{b}}{4 \Omega_{r}}\right)^{1 / 2} E(z)} .
$$


This distance represents the maximum distance over which the baryon oscillations imprint maximum fluctuations in thermal radiation that is observed as anisotropies in CMB. This represents an angular size $\theta_{\text {sh }}$ observed at an angular diameter distance $d_{A}\left(z_{l s}\right)$ given simply by

$$
D_{A}\left(z_{l s}\right) \equiv S_{k}\left(d_{P}\right) /\left(1+z_{l s}\right), \text { and } \theta_{s h} \equiv d_{s h} / D_{A}
$$

And the corresponding multipole moment is given by $l=\pi / \theta_{s h}$.

Next thing is to determine the correct value of $z_{l s}$ corresponding to the last scattering surface when the physical constants are varying. The blackbody radiation energy density $\varepsilon_{\gamma}$ is given by ${ }^{29} \varepsilon_{\gamma}=$ $8 \pi^{2} k^{4} T^{4} / 15 h^{3} c^{3}$ ( $k$ being the Boltzmann constant), and the radiation energy density as per Eq. (10) is given by $\varepsilon_{r}=\varepsilon_{0} a^{-4}$. Therefore, when the constants $k, h$ and $c$ are invariant then $T \propto a^{-1}$ with $a^{-1}=1+$ $z$. Using the fact that the $h$ varies as $c$, and $k$ varies as $c^{1.25}$ as presented in Section 4 of this paper, we see that $T \propto a^{-1} \exp \left[0.25\left(a^{\alpha}-1\right)\right]$. Additionally, we have to see how does the ionization energy $Q$ of an atom evolve since it is proportional to $R_{\infty} h c$, where $R_{\infty} \equiv m_{e} e^{4} / 8 \epsilon_{0}^{2} h^{3} c$ with $m_{e}$ the mass of electron, $e$ its charge and $\epsilon_{0} \propto 1 / c^{2}$ the permittivity of space. Thus $R_{\infty}$ is invariant and therefore $Q \propto h c$. And then how does the thermal photon energy $k T$ evolve, as the ratio of the ionization energy and thermal energy determines the temperature of the last scattering surface? This ratio can be seen to evolves as $(h c / k)$ $\propto \exp \left[0.75\left(a^{\alpha}-1\right)\right]$. Cumulatively, the two effect lead to $T \propto a^{-1} \exp \left[\left(a^{\alpha}-1\right)\right] \equiv a^{\prime-1}$. Now $\exp [(1+$ $\left.z)^{-\alpha}-1\right]=1 / e$ for $z \gg 1$. Therefore $T=T_{0} a^{\prime-1}=T_{0}(a e)^{-1}$. Thus $a^{\prime-1}=1+z^{\prime}=(1+z) / 2.7183$. We can now see that when $z=1089, z^{\prime}=400$, i.e. the last scattering surface is at the redshift 400 in our VPC models.

One may question that the blackbody spectrum will be affected due to the varying physical constants since the energy density for the photons in the frequency range $v$ and $v+d v$ is given by $\varepsilon(v) d v=$ $\frac{8 \pi h v^{3}}{c^{3}}\left[d v /\left(\exp \left(\frac{h v}{k T}\right)-1\right)\right]$, which would evolve due to the variation of $c, h$ and $k$. However, we do not know what exactly the spectrum of the distant cosmological objects is? We only know what it is when it is observed. If we consider the peak photon energy of the spectrum, it is given by ${ }^{29} h v_{\max }=2.8 \mathrm{kT}$, or $v_{\text {max }}=\frac{2.8 k}{h} T=\frac{2.8 k_{0}}{h_{0}} T_{0} \exp \left[\frac{1}{4}\left(a^{1.8}-1\right)\right]$. Then $\dot{v}_{\max }=\frac{2.8 k_{0}}{h_{0}} T_{0} \exp \left(0.25\left(a^{1.8}-1\right)\right) \times 0.45 a^{1.8}\left(\frac{\dot{a}}{a}\right)$. Therefore $\frac{\dot{v}_{\max }}{v_{\max }}=0.45 a^{1.8} \dot{a} / a$, which, near $a=a_{0}$, i.e. $t=t_{0}$ is $\frac{\dot{v}_{\max }}{v_{\max }}=0.45 H_{0}$. Since $H_{0} \approx 71 \mathrm{~km} \mathrm{~s}^{-1} \mathrm{Mpc}^{-1}=2.3 \times$ $10^{-18} s^{-1}$, we get $\frac{\dot{v}_{\max }}{v_{\max }} \approx 1 \times 10^{-18} s^{-1}$. This is very small shift to observe in the blackbody spectrum. Nevertheless, it would be nice if one could come up with some observation or experiment which could detect the thermal spectrum variation with time.

\subsection{Other Cosmological Parameters}

Let us now consider some other cosmological parameters.

Deceleration Parameter: The second Friedmann equation, Eq. (7) is used to determine the deceleration parameter $q_{0}$, which by definition ${ }^{31}$

$$
q_{0}=-\left(\frac{\ddot{a}}{a H^{2}}\right)_{t=t_{0}}=-\frac{1}{H_{0}^{2}}\left(\frac{\ddot{a}}{a}\right)_{t=t_{0}} .
$$

Recalling that $a_{0}=1$ and $\dot{c} / c=\alpha H_{0}$ in the limit of $t=t_{0}$, we may write Eq. (7) at $t=t_{0}$ as

$$
\begin{gathered}
\left(\frac{\ddot{a}}{a}\right)_{t=t_{0}}=-\frac{4 \pi G_{0}}{3 c_{0}^{2}} \varepsilon_{0}(1+3 w)+\frac{\Lambda_{0} c_{0}^{2}}{3}+H_{0}\left(\frac{\dot{c}}{c}\right)_{t=t_{0}}, \text { or } \\
q_{0}=-\frac{\ddot{a}_{0}}{H_{0}^{2}}=\frac{8 \pi G_{0}}{3 c_{0}^{2} H_{0}^{2}}\left[\varepsilon_{0}\left(\frac{1}{2}+\frac{3}{2} w\right)-\frac{\Lambda_{0} c_{0}^{4}}{8 \pi G_{0}}\right]-\alpha .
\end{gathered}
$$


Since $\varepsilon_{c, 0}=\frac{8 \pi G_{0}}{3 c_{0}^{2} H_{0}^{2}}$, we get

$$
q_{0}=\frac{1}{2} \Omega_{m, 0}+\Omega_{r, 0}-\Omega_{\Lambda, 0}-\alpha
$$

The last term results from the varying physical constants approach.

Age of the Universe: We need to calculate the parameter $t_{0}$ that is the current cosmic time relative to the big-bang time. From Eq. (18), for VcG $\Lambda$ model

$$
\begin{gathered}
\frac{H}{H_{0}}=E(a) \Rightarrow \frac{\dot{a}}{a}=H_{0} E(a) \Rightarrow \frac{d a}{d t}=H_{0} a E(a) \Rightarrow H_{0} d t=\frac{d a}{a E(a)} \equiv \frac{d a}{F(a)} \\
F(a)^{2}=\exp \left[\left(a^{\alpha}-1\right)\right]\left(\Omega_{m, 0} a^{-1}+\Omega_{r, 0} a^{-2}\right)\left(1+\frac{\alpha}{\beta} a^{\alpha-\beta}\right)+\Omega_{k, 0} \exp \left[2\left(a^{\alpha}-1\right)\right] \\
\therefore t_{0}=\int_{0}^{t_{0}} d t=\frac{1}{H_{0}} \int_{0}^{1} d a / F(a) .
\end{gathered}
$$

BAO Acoustic Scale: It relates to baryon acoustic oscillations (BAO) that are linked directly to the CMB anisotropies. BAO is considered observable today through the correlation function of galaxies' distribution in space ${ }^{32}$. It is given by:

$$
r_{a s}=d_{s h}\left(z_{l s}\right)\left(1+z_{l s}\right)
$$

Here $d_{s h}$ is given by Eq. (35) and $z_{l s}=400$ for the VPC models. This parameter could be considered another test for the proposed VPC model.

\section{Results}

We will now test the proposed model against observations. The most used test is to see how a model fits the redshift - distance modulus $(z-\mu)$ data from the observation on supernovae 1a (standard candle). The data used in this work is the so-called Pantheon Sample ${ }^{24}$ of 1048 supernovae Ia in the range of $0.01<z<2.3$. The data is in terms of the apparent magnitude and we added 19.35 to it to obtain normal distance modulus numbers as suggested by Scolnic ${ }^{33}$.

The Matlab curve fitting tool was used to fit the data by minimizing $\chi^{2}$ and the latter was used for determining the corresponding $\chi^{2}$ probability ${ }^{34} P$. Here $\chi^{2}$ is the weighted summed square of residual of $\mu$

$$
\chi^{2}=\sum_{i=1}^{N} w_{i}\left[\mu\left(z_{i} ; H_{0}, p_{1}, p_{2} \ldots\right)-\mu_{o b s, i}\right]^{2},
$$

where $N$ is the number of data points, $w_{i}$ is the weight of the $i$ th data point $\mu_{o b s, i}$ determined from the measurement error $\sigma_{\mu_{O b s, i}}$ in the observed distance modulus $\mu_{o b s, i}$ using the relation $w_{i}=1 / \sigma_{\mu_{O b s, i}}^{2}$ and $\mu\left(z_{i} ; H_{0}, p_{1}, p_{2} ..\right)$ is the model calculated distance modulus dependent on parameters $H_{0}$ and all other model dependent parameter $p_{1}, p_{2}$, etc. As an example, for the $\Lambda \mathrm{CDM}$ models considered here, $p_{1} \equiv \Omega_{m, 0}$ and there is no other unknown parameter.

We then quantified the goodness-of-fit of a model by calculating the $\chi^{2}$ probability for a model whose $\chi^{2}$ has been determined by fitting the observed data with known measurement error as above. This probability $P$ for a $\chi^{2}$ distribution with $n$ degrees of freedom (DOF), the latter being the number of data points less the number of fitted parameters, is given by: 


$$
P\left(\chi^{2}, n\right)=\left(\frac{1}{\Gamma\left(\frac{n}{2}\right)}\right) \int_{\chi^{2} / 2}^{\infty} e^{-u} u^{\frac{n}{2}-1} d u
$$

where $\Gamma$ is the well know gamma function that is generalization of the factorial function to complex and non-integer numbers. The lower the value of $\chi^{2}$, the better the fit, but the real test of the goodness-of-fit is the $\chi^{2}$ probability $P$; the higher the value of $P$ for a model, the better the model's fit to the data. We used an online calculator to determine $P$ from the input of $\chi^{2}$ and DOF ${ }^{35}$.

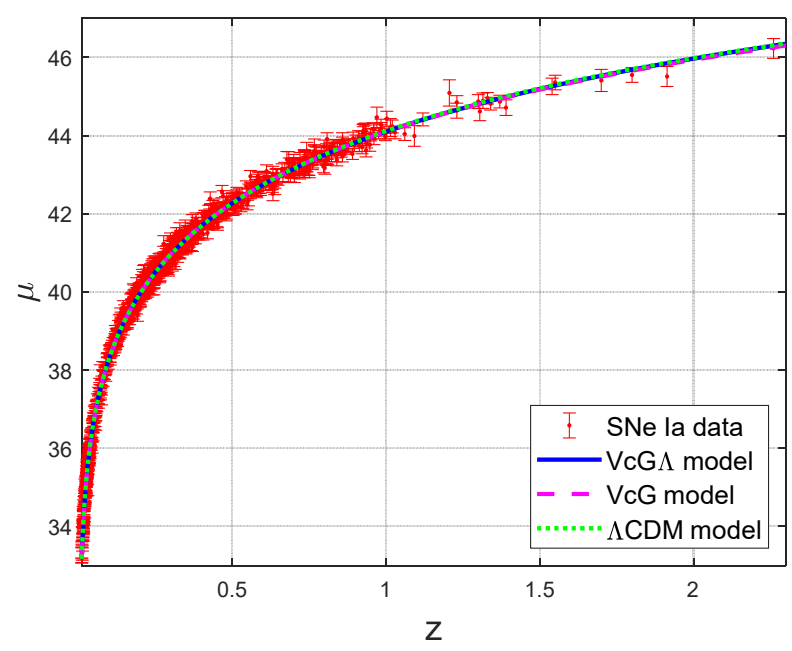

Fig. 1. Supernovae Ia redshift $z$ vs. distance modulus $\mu$ data fits using the VcG $\Lambda$ model and VcG model as compared to the fit using the $\Lambda \mathrm{CDM}$ model. All the curves are so close to each other that they appear to be superimposed.

Table 1. Parameters for the three models determined by fitting the Pantheon Sample SNe Ia $z-\mu$ data. This table shows all the models are able to fit the data very well with the VcG $\Lambda$ model slight edge on others. The unit of $\mathrm{H}_{0}$ is $\mathrm{km} \mathrm{s}^{-1} \mathrm{Mpc}^{-1}$. $\mathrm{P} \%$ is the $\chi 2$ probability in percent; the higher the $\chi 2$ probability $P$, the better the model fits to the data. $R^{2}$ is the square of the correlation between the response values and the predicted response values. RMSE is the root mean square error and DOF is the degrees of freedom.

\begin{tabular}{|c|c|c|c|}
\hline Parameter & $\Lambda \mathrm{CDM}$ & $\mathrm{VcG}$ & $\mathrm{VcG} \Lambda$ \\
\hline $\mathrm{H}_{0}$ & $70.18 \pm 0.42$ & $70.79 \pm 0.70$ & $70.87 \pm 0.50$ \\
\hline$\Omega_{\mathrm{m}, 0}$ & $0.2845 \pm 0.0245$ & $1.053 \pm 0.1363$ & $0.528 \pm 0.0 .037$ \\
\hline$\Omega_{\Lambda, 0}$ & $1-\Omega_{\mathrm{m}, 0}$ & $0.06441 \pm 0.22419$ & $-\Omega_{\mathrm{m}, 0}$ \\
\hline$\Omega_{0}$ & 1 & 1.117 & 0 \\
\hline$\alpha$ & $\mathrm{NA}$ & 1.25 & 1.8 \\
\hline$\beta$ & $\mathrm{NA}$ & $\mathrm{NA}$ & -1.8 \\
\hline$\chi^{2}$ & 1036 & 1032 & 1032 \\
\hline DOF & 1046 & 1045 & 1046 \\
\hline P\% & 58.1 & 60.7 & 61.5 \\
\hline R-sq & 0.997 & 0.997 & 0.997 \\
\hline RMSE & 0.9951 & 0.9938 & 0.9933 \\
\hline
\end{tabular}


Our primary findings are presented in Figure 1 and Table 1.

One additional thing we wished to explore was if the prior of $\sigma=3$ is the right choice for the VcG $\Lambda$ model. This can be confirmed by fitting the SNe Ia data. Thus, with all the parameters $\left(H_{0}, \Omega_{m, 0}, \alpha, \beta, \sigma\right)$ free, i.e. no priors, we obtained results closed to the values in Table 1 but with large $95 \%$ confidence bounds. When we progressively constrained the parameters based on our prior knowledge, the 95\% confidence bounds shrank and we obtained those parameters still unconstrained as expected. The results are presented in Table 2 .

Table 2. This table shows the results of fitting the Pantheon Sample SNe Ia $z-\mu$ data in order to determine the value of the parameter $\sigma$ that is used in the VcG $\Lambda$ model. The description of other parameters is the same as in Table 1. The unit of $\mathrm{H}_{0}$ is $\mathrm{km} \mathrm{s}^{-1} \mathrm{Mpc}^{-1}$.

\begin{tabular}{|l|c|c|c|c|}
\hline Parameter & All parameters free & 4 parameters free & 3 parameters free & 2 parameters free \\
\hline $\mathrm{H}_{0}$ & $70.47_{+1200}^{-1059}$ & 70.87 Fixed & 70.87 Fixed & 70.87 Fixed \\
\hline$\Omega_{\mathrm{m}, 0}$ & $0.5273_{+21.91}^{-20.86}$ & $0.5211_{0.6857}^{0.356}$ & $0.5298_{0.7058}^{0.3537}$ & $0.528_{0.5575}^{0.4989}$ \\
\hline$\alpha$ & $1.85_{+39.64}^{-35.94}$ & $1.854_{2.938}^{0.769}$ & 1.8 Fixed & 1.8 Fixed \\
\hline$\beta$ & $-2.29_{+121.4}^{-125.9}$ & $-2.204_{+10.39}^{-14.8}$ & $-1.756_{+2.189}^{-5.701}$ & -1.8 Fixed \\
\hline$\sigma$ & $3.027_{+77.22}^{-71.17}$ & $3.001_{3.059}^{2.942}$ & $3.001_{3.045}^{2.956}$ & $3_{3.028}^{2.973}$ \\
\hline$\chi^{2}$ & 1032 & 1032 & 1032 & 1032 \\
\hline DOF & 1043 & 1044 & 1045 & 1046 \\
\hline P\% & 58.98 & 59.83 & 60.67 & 61.51 \\
\hline R-sq & 0.997 & 0.997 & 0.997 & 0.997 \\
\hline RMSE & 0.9948 & 0.9943 & 0.9938 & 0.9933 \\
\hline
\end{tabular}

We notice that as the parameters are progressively fixed, the $\chi^{2}$ value does not change. This means that the constraint put on the parameter is reasonable, otherwise $\chi^{2}$ value would have increased. In fact, the $\chi^{2}$ probability $P$ slightly improves and so does the RMSE. SNe Ia data fit therefore confirms our choice of $\sigma=3$ and $\alpha=1.8$ for the $\operatorname{VcG} \Lambda$ model based on our previous work.

The SNe Ia test is to see how good a model is for the directly observable universe for which the redshift and light fluxes of galaxies are measurable. This test is the primary test. If the model fails this test then it may not be worthwhile to consider it for other tests.

The second test is to see how well the model computes the multipole moment of the first peak in the power spectrum due to the temperature anisotropies in the cosmic microwave background. WMAP (Wilkinson Microwave Anisotropy Probe) and Plank ${ }^{36}$ have determined the multipole moment $l \approx 220$ for the first peek. A third test may be considered how well the models match the value of the BAO acoustic scale and compare it with the measured ${ }^{32}$ and Planck $^{36}$ estimate of this parameter. To be consistent with the measured value using Hubble constant ${ }^{32}$ of $H_{0}=70 \mathrm{~km} \mathrm{~s}^{-1} \mathrm{Mpc}^{-1}$, we have normalized the two other models to $H_{0}=70 \mathrm{~km} \mathrm{~s}^{-1} \mathrm{Mpc}^{-1}$ for computing BAO acoustic scale only.

Another important test comprises determining the age of the universe. We have determined it for the models and compared it with the well accepted value of $13.7 \mathrm{Gyr}$.

Table 3 presents the above tests. It also includes deceleration parameter, $q_{0}$, as well as some other cosmological parameters for ready comparison. Both the VPC models estimate higher acceleration than the $\Lambda$ CDM model. 
Table 3. This table presents the results of other tests for the two models and compare them to the $\Lambda C D M$ fit derived from the CMB temperature anisotropy observations by Planck [36]. The Hubble constant used for the other two models is the same as obtained for these models from SNe Ia data fit. VSL stands for varying speed of light. The unit of $\mathrm{H}_{0}$ is $\mathrm{km} \mathrm{s}^{-1} \mathrm{Mpc}^{-1}$.

\begin{tabular}{|c|c|c|c|c|}
\hline \multicolumn{2}{|c|}{ Parameter } & $\begin{array}{c}\text { Planck+BAO } \\
\text { NCDM [36] }\end{array}$ & VcG & VcG $\Lambda$ \\
\hline Matter energy density & $\Omega_{\mathrm{m}, 0}$ & 0.3106 & 1.053 & 0.528 \\
\hline Dark-energy density & $\Omega_{\Lambda, 0}$ & 0.6894 & 0.06441 & -0.528 \\
\hline Curvature parameter & $\Omega_{\mathrm{k}, 0}$ & -0.0096 & -0.1175 & 1.0000 \\
\hline Hubble constant & $\mathrm{H}_{0}$ & 67.70 & 70.79 & 70.87 \\
\hline Redshift at last scattering surface & $\mathrm{z}$ & 1090 & 1090 & 1090 \\
\hline VSL corrected redshift (see text) & $\mathrm{z}^{\prime}$ & 1090 & 400 & 400 \\
\hline 1st peak's multipole moment & $l$ & 220.6 & 130 & 217.3 \\
\hline Deceleration parameter & $\mathrm{q}_{0}$ & -.5341 & -0.788 & -1.01 \\
\hline Age of the universe & $\mathrm{t}_{0}(\mathrm{Gyr})$ & 13.787 & 6.068 & 14.108 \\
\hline $\begin{array}{c}\text { BAO acoustic scale (measured } \\
\text { value 149.28) }\end{array}$ & $\mathrm{r}_{\mathrm{s}}(\mathrm{Mpc})$ & 147.57 & 180.6 & 145.2 \\
\hline
\end{tabular}

\section{Discussion}

An objective of this paper is to see if Costa et al.'s22 theory complements our quasi-phenomenological model and provides improvement on it by being covariantly relativistic. The resulting Friedmann equations and continuity equation eliminates the arbitrariness of our earlier models ${ }^{21,28}$. The continuity equation, Eq. (8) essentially breaks down into three separate equations: the first for energy density $\varepsilon$, the second relating speed of light $c$ to the gravitational constant $G$, and the third relating the energy density $\varepsilon$ to the dark-energy density through the cosmological constant $\Lambda$. As a result the solution of the Freedmann equations is greatly simplified even though the physical constants $c, G$ and $\Lambda$ are allowed to vary. Despite their simplicity, the VcG $\Lambda$ model they yield is highly satisfactory in explaining the observations from $z=0$ to $z=1100$.

The first test both the VcG and VcG $\Lambda$ models passed is the SNe 1a test (which involves relatively low redshift data) as is clear from studying Table 1 and Figure 1 . However, as is obvious from Table 3 , the $\mathrm{VcG}$ model badly failed all other tests including the age of the universe test and those which involve high redshift observations whereas VcG $\Lambda$ model passed them all with flying colors. Few noticeable things about the VcG $\Lambda$ model in the table are: (a) the sign of the dark-energy density is negative, (b) the curvature parameter $\Omega_{k, 0}=1$ is rather high relative to almost negligible value for the $\Lambda$ CDM model, meaning that the space is curved negatively, and (c) the deceleration parameter is almost twice as much as for the $\Lambda \mathrm{CDM}$ model meaning that the universe's expansion is accelerating at almost twice the rate predicted by the $\Lambda \mathrm{CDM}$ model. These discrepancies are a result of the varying physical constants.

Thus the staggering feature of the varying physical constant model VcG $\Lambda$ is that it yields negative dark-energy density while still showing accelerated expansion of the universe. Possibility of such a scenarios has been explored by many researchers ${ }^{37-40}$ who considered the varying cosmological constant to primarily resolve Hubble constant tension, which is due to the difference in its values obtained from $\mathrm{CMB}$ data and SNe Ia data. Such a scenario is extremely interesting from the string theory perspective as obtaining a vacuum solution with a positive value of $\Lambda$ within moduli-fixed consistent and stable string theory compactifications has been a formidable task ${ }^{41-44}$. 
It is even more staggering that the spatial curvature of the universe is strongly negative rather than flat and thus contradicts all the theories, and observations derived assuming invariant physical constants. When analysing any observational data we need to be careful that it is not biased in favour of the physical constants that are constrained to their currently observed values, especially in view of the new definition of length dependent on the constant value of the speed of light as 299,792,458 meters per second ${ }^{45}$. As discussed below, physical constants other than $c, G$ and $\Lambda$ that are not directly involved in the models here, such as Planck constant $h$ and Boltzmann constant $k$ also vary. This makes it even more difficult to reach conclusions based on observations that directly or indirectly involve many physical constants.

It should also be pointed out that the matter density $\Omega_{m, 0}=0.528$, obtained by VcG $\Lambda$ model is significantly higher than that estimated by the Planck mission $\Omega_{m, 0}=0.3153$ assuming $\Lambda$ CDM cosmology ${ }^{36}$. The Planck mission also estimated the baryon density $\Omega_{b, 0}=0.049$ and dark-matter density $\Omega_{d m, o}=0.2607$ (Planck papers label it as $\Omega_{c}$ ). Since the baryon density has also been estimated directly by accounting the visible and non-visible baryonic matter, we can assume it to be valid for any cosmological model. Then the dark-matter density for the VcG $\Lambda$ model $\Omega_{d m, o}=0.479$ is $84 \%$ higher than the $\Lambda$ CDM model. Cosmologist now will have more freedom to play with the dark-matter.

The results presented here may be considered a continuation of our previous work ${ }^{21}$ wherein we explained three astrometric anomalies and the null results on the variation of $G$ and the fine structure constant using the quasi-phenomenological version of the current $\mathrm{VcG} \Lambda$ model. Since our current model reduces to the quasi-phenomenological model in the limit of the scale factor $a$ close to its current value 1, and since all these problems relate to the space where scale factor $a$ is close to 1 , we conclude that the current model is also able to resolve these problems. Thus the findings of our previous work that the Planck constant $h$ varies just like the speed of light $c$, inferred from the null results on the variation of the fine-structure constant $\alpha$, can also be considered valid under the VcG $\Lambda$ model.

We have assumed in our work here that $k$ varies as $c^{1.25}$. This assumption lead to the redshift $z^{\prime}(=$ $z / e$ ) for the surface of last scattering to be 400 . It is confirmed from our results for the first peak of the $\mathrm{CMB}$ power spectrum and $\mathrm{BAO}$ acoustic scale which are within $3 \%$ of the standard $\Lambda \mathrm{CDM}$ model and observations. One could be sure that the results would only improve with the refinement of the model.

It would be interesting to see how some of the well-known constants vary when the fundamental constants are considered evolutionary. Consider for example the Rydberg constant $R_{\infty}=\frac{m_{e} e^{4}}{8 \varepsilon_{0}^{2} h^{3} c}$ where $m_{e}$ is the rest mass of the electron, $e$ is the elementary charge, and $\varepsilon_{0}$ is the permittivity of space. If we consider $m_{e}$ and $e$, which belong to baryonic matter, to be invariant (at least in comparison to the variation of the fundamental constants considered here), and since $\varepsilon_{0} \propto 1 / c^{2}$ and $h$ varies as $c$, we infer that $R_{\infty}$ is invariant. Similarly, the fine-structure constant, given by $\alpha=\frac{1}{4 \pi \varepsilon_{0}} \frac{e^{2}}{\hbar c}$ will be immune to the variation of $h$ and $c$. As already mentioned, it is the constancy of the fine-structure constant that led us to conclude in our previous work ${ }^{21}$ that the Planck constant varies as the speed of light. Any variations in these constant would mean either our assumptions are wrong or $e$ and $m_{e}$ do vary to the extent of the measured variations in the Rydberg constant and the fine structure constant.

\section{Conclusions}

We have shown that the VcG $\Lambda$ model, based on the covariant relativistic approach for including variation of fundamental physical constants can explain, with very few parameters, the cosmological observation considered in this work, as well or better than the standard $\Lambda$ CDM model. Most difficult to accept findings of the current work are (a) the evolutionary dark-energy density being negative, and (b) the universe having significant negative spatial curvature. The VcG $\Lambda$ model (i) fits the supernovae Ia observational data slightly better than the $\Lambda \mathrm{CDM}$ model; (ii) determines the first peak in the power spectrum of the cosmic microwave background temperature anisotropies at the multipole value of 
$l=217.3$ against the observed value of 220; (iii) calculates the age of the universe as 14.1 Gyr against the accepted value of $13.7 \mathrm{Gyr}$; and (iv) finds the BAO acoustic scale to be $145.2 \mathrm{Mpc}$ against the observed value of 149.3. We therefore conclude that the VcG $\Lambda$ model deserves to be considered seriously for further work which needs collaboration to study more difficult cosmological problems such as fitting the power spectrum of CMB temperature anisotropies. Current codes such as $\mathrm{CAMB}^{46}, \mathrm{CLASS}^{47}$ and $\mathrm{CMBAns}^{48}$ are unable to handle background based on VPC models and it is challenging undertaking to modify them ${ }^{49,50 .}$

\section{Acknowledgements}

This work has been supported by a generous grant from Macronix Research Corporation. Thanks are due to Madhav Singhal at the University of Western Ontario who used his python code to verify the SNe 1a data fit by Matlab curve fitting app. The author is grateful to Krishnakanta Bhattacharya at the Indian Institute of Technology, Guwahati, for his critical comments and helpful discussions. He remains thankful to Dan Scolnic at Duke University for providing the SNe 1a Pantheon Sample data used in this work, and to Santanu Das at the University of Wisconsin, Madison, for his comments and discussion on the use and modification of CMB codes.

\section{References}

1. W. Thomson and P. G. Taid, Treatise on natural philosophy (Cambridge University Press, 1883).

2. H. Weyl, Ann. Phys. 59 (1919) 129.

3. A. S. Eddington, New Pathways in Science (Cambridge University Press, 1934).

4. P. A. M. Dirac, Nature 139 (1937) 323.

5. P. A. M. Dirac, Proc. R. Soc. A 65 (1938) 199.

6. C. Barns and R. H. Dicke, Phys. Rev. 124 (1961) 925.

7. A. Einstein, Jahrbuch fur Radioaktivitat und Elektronik 4, (1907) 11.

8. R. H. Dicke, Rev. Mod. Phys. 29 (1957) 363.

9. J.-P. Petit, Mod. Phys. Lett. A 3 (1988) 1527; ibid 1733; ibid 2201.

10. J. W. Moffat, Int. J. Mod. Phys. D 2 (1993) 351.

11. J. W. Moffat, Found. Phys. 23 (1993) 411.

12. A. Albrecht and J. Magueijo, Phys. Rev. D 59 (1999) 043516.

13. J. D. Barrow, Phys. Rev. D 59 (1999) 043515.

14. P. P. Avelino and C. J. A. P. Martins, Phys. Lett. B 459 (1999) 468.

15. P. P. Avelino, C. J. A. P. Martins and G. Rocha, Phys. Lett. B 483 (2000) 210.

16. J. W. Moffat, Eur. Phys. J. C 76 (2016) 130.

17. J.-P. Uzan, Rev. Mod. Phys. 75 (2003) 403.

18. J.-P. Uzan, Living Rev. Relativ. 14 (2011) 2.

19. T. Chiba, T. Prog. Theor. Phys. 126 (2011) 993.

20. G. F. R. Ellis and J.-P.Uzan, Am. J. Phys. 73 (2005) 240.

21. R. P. Gupta, Galaxies 7 (2019) 55.

22. R. Costa, R. R. Cuzinatto, E. G. M. Ferreira and G. Franzmann, Int. J. Mod. Phys. D 28 (2019) 1950119.

23. G. Franzmann, arXiv:1704.07368 (2017).

24. D. M. Scolnic, D. O. Jones, A. Rest, Y. C. Pan, R. Chornock, R. J. Foley, M. E. Huber, R. Kessler, G.

Narayan, A. G. Riess, et al., Astrophys. J. 859 (2018) 101.

25. Maple ver. 2019.2, Maplesoft, Waterloo, Canada (2019).

26. J. D. Barrow and J. Magueijo, Phys. Lett. B 447 (1999) 246.

27. V. Salzano and M. P. Dabrowski, Astrophys. J. 851 (2017) 97.

28. R. P. Gupta, Universe 4 (2018) 104.

29. B. Ryden, Introduction to Cosmology (Cambridge University Press, 2017). 
30. R. Durrer, The Cosmic Microwave Background (Cambridge University Press, 2008) p. 211.

31. J. V. Narlikar, An Introduction to Cosmology, $3^{\text {rd }}$ Ed. (Cambridge University Press, 2002).

32. L. Anderson, É. Aubourg, S. Bailey, F. Beutler, V. Bhardwaj, M. Blanton, A. S. Bolton, J. Brinkmann, J. R. Brownstein, A. Burden, et al., Mon. Not. Roy. Astron. Soc. 441 (2014) 24.

33. D. M. Scolnic, personal email communication (November 22, 2018).

34. W. H. Press, S. A. Teukolsky, W. T. Vetterling and B. P. Flannery, Numerical Recipes in C-The Art of Scientific Computing, 2nd ed. (Cambridge University Press, 1992).

35.J. Walker, Chi-Square Calculator: https://www.fourmilab.ch.rpkp/experim-ents/analysis/chiCalc.html (accessed on 20 June 2020).

36. Planck Collaboration, N. Aghanim, Y. Akrami, M. Ashdown, J. Aumont, C. Baccigalupi, et al., Planck 2018 results. I. Overview and cosmological legacy of Planck, (Astron. \& Astrophys. Oct. 25 , 2019).

https://www.aanda.org/articles/aa/pdf/forth/aa33880-18.pdf; https://arxiv.org/abs/1807.06205v2.

37. L. Visinelli, S. Vagnozzi and U. Danielsson, Symmetry 11 (2019) 1035.

38. K. Dutta, A. Roy, Ruchika et al., Gen. Relativ. Gravit. 52 (2020) 15.

39. J. B. Hartle, S. W. Hawking and T. Hertog, Accelerated Expansion From Negative $\Lambda$, arXiv:1205.3807.

40. Y. Wang, L. Pogosian, G.-B. Zhao and A. Zucca, Astrophys. J. Lett. 869 (2018) L8.

41. J. M. Maldacena and C. Nunez, Int. J. Mod. Phys. A 16 (2001) 822.

42. S. Kachru, R. Kallosh, A. D. Linde and S. P. Trivedi, Phys. Rev. D 68 (2003) 046005.

43. J. P. Conlon and F. Quevedo, J. Cosmo. Astropart. Phys. AP 0708 (2007) 019.

44. U. H. Danielsson and T. Van Riet, Int. J. Mod. Phys. D 27 (2018) 1830007.

45. NIST https://www.nist.gov/pml/weights-and-measures/si-units-length.

46. A. Lewis, A. Challinor and A. Lasenby, Astrophys. J. 538 (2000) 473.

47. J. Lesgourgues, The cosmic linear anisotropy solving system (class) I: Overview, arXiv:1104.2932.

48.S. Das and A. Phan, Cosmic Microwave Background Anisotropy numerical solution (CMBAns) I: An introduction to $\mathrm{Cl}$ calculation, axXiv: 1910.00725.

49. A. Lewis, personal email communication (May, 2020 ).

50.S. Das, personal email communication and discussions (Jan-June, 2020). 